\title{
Antireflux versus conventional self-expanding metallic Stents (SEMS) for distal esophageal cancer: results of a multicenter randomized trial
}

Authors

Institutions
E. Coron ${ }^{1,2, *}$, G. David ${ }^{1,2, *}$, S. Lecleire ${ }^{3}$, J. Jacques ${ }^{4}$, A. Le Sidaner ${ }^{4}$, T. Barrioz ${ }^{5}$, D. Coumaros ${ }^{6}$, C. Volteau $^{7}$, B. Vedrenne ${ }^{8}$, P. Bichard ${ }^{9}$, C. Boustière ${ }^{10}$, Y. Touchefeu ${ }^{1,2}$, J. Brégeon ${ }^{2}$, F. Prat ${ }^{11}$, M. Le Rhun ${ }^{1,2}$ and the Société Française d'Endoscopie Digestive (SFED)

Institutions are listed at the end of article. submitted 23. October 2015 accepted after revision 18. January 2016

\section{Bibliography}

Dol http://dx.doi.org/

10.1055/s-0042-106960

Endoscopy International Open

2016; 04: E730-E736

(c) Georg Thieme Verlag KG

Stuttgart · New York

E-ISSN 2196-9736

Corresponding author

Pr Emmanuel Coron

Institut des Maladies de

I'Appareil Digestif

CHU Hotel Dieu

1 Place Alexis Ricordeau

44093 Nantes Cedex

France

emmanuel.coron@chu-nantes. $\mathrm{fr}$
Introduction: Self-expanding metal stents (SEMS) are commonly used in the palliation of dysphagia in patients with inoperable esophageal carcinoma. However, they predispose to gastroesophageal reflux when deployed across the gastroesophageal junction. The aims of this study were to: 1 ) assess the influence of the antireflux valve on trans-prosthetic reflux (primary outcome); and 2) compare the results of SEMS with and without antireflux valve in terms of reflux symptoms, quality of life (QOL), improvement of dysphagia and adverse events (secondary outcomes).

Patients and methods: Thirty-eight patients were enrolled in nine centers. Carcinomas were locally advanced (47\%) or metastatic. After randomization, patients received either a covered SEMS with antireflux valve $(n=20)$ or a similar type of SEMS with no antireflux device but assigned to standard proton pump inhibitor therapy and postural advice $(n=18)$. Trans-prosthetic reflux was assessed at day 2 using a radiological score based on barium esophagography performed after Trendelenburg maneuver and graded from 0 (no reflux) to 12 (maximum). Monthly telephone interviews were conducted for Organisation Mondiale de la Santé (OMS) scoring from 0 (excellent) to 5 (poor), QOL assessment (based on the Reflux-

\section{Introduction}

$\nabla$

Dysphagia is the most frequent symptom in patients presenting with an esophageal or gastric cardia cancer. Due to the late occurrence of symptoms, the goal of management in such cancers is focused on palliation in more than half of cases. In addition, the incidence of esophageal cancer is rising and the prognosis is poor with a 5-year overall survival rate less than $10 \%$, which emphasizes the importance of palliative treatments [1].

\section{License terms}

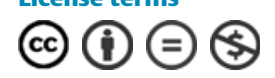

* These authors contributed equally.
Qual Simplifié scoring system) from 0 (poor) to 100 (excellent), dysphagia scoring from 0 (no dysphagia) to 5 (complete dysphagia) and regurgitation scoring from 0 (no regurgitation) to 16 (maximum).

Results: No difference was noted in terms of age, sex, size of lesion, prosthesis length or need for dilation prior to SEMS placement. No difficulty in placing SEMS nor complications were noted. Radiological scores of reflux were found to be significantly lower in patients with an antireflux stent compared to the conventional stent and associated measures. The regurgitation scores were significantly decreased in patients with antireflux stents during the first 2 months after stent placement and thereafter, they were similar in the two groups. QOL and dysphagia were improved in both groups. Survival rates were comparable in the two groups.

Conclusions: No difference was observed between the two types of SEMS regarding the palliation of dysphagia and improvement of QOL. However, SEMS with an antireflux valve were more effective in preventing trans-prosthetic gastroesophageal reflux but at the cost of an increased likehood of minor adverse events (migrations and/or obstruction of the SEMS).

Indeed, relief of dysphagia is a major issue in these situations, since it is responsible for poor quality of life, under nutrition, and performance status alteration [2,3]. Insertion of a self-expanding metal stent (SEMS) relieves malignant dysphagia and is associated with an improvement in patient' quality of life [4-7]. Extension of adenocarcinoma of the distal esophagus frequently involves the gastro-esophageal junction. Therefore, deployment of SEMS in this location results in positioning the lower extremity of the stent in the stomach. While this position does not impair the efficacy of the stent in palliation of dysphagia, it has two major drawbacks: first, it increases the 

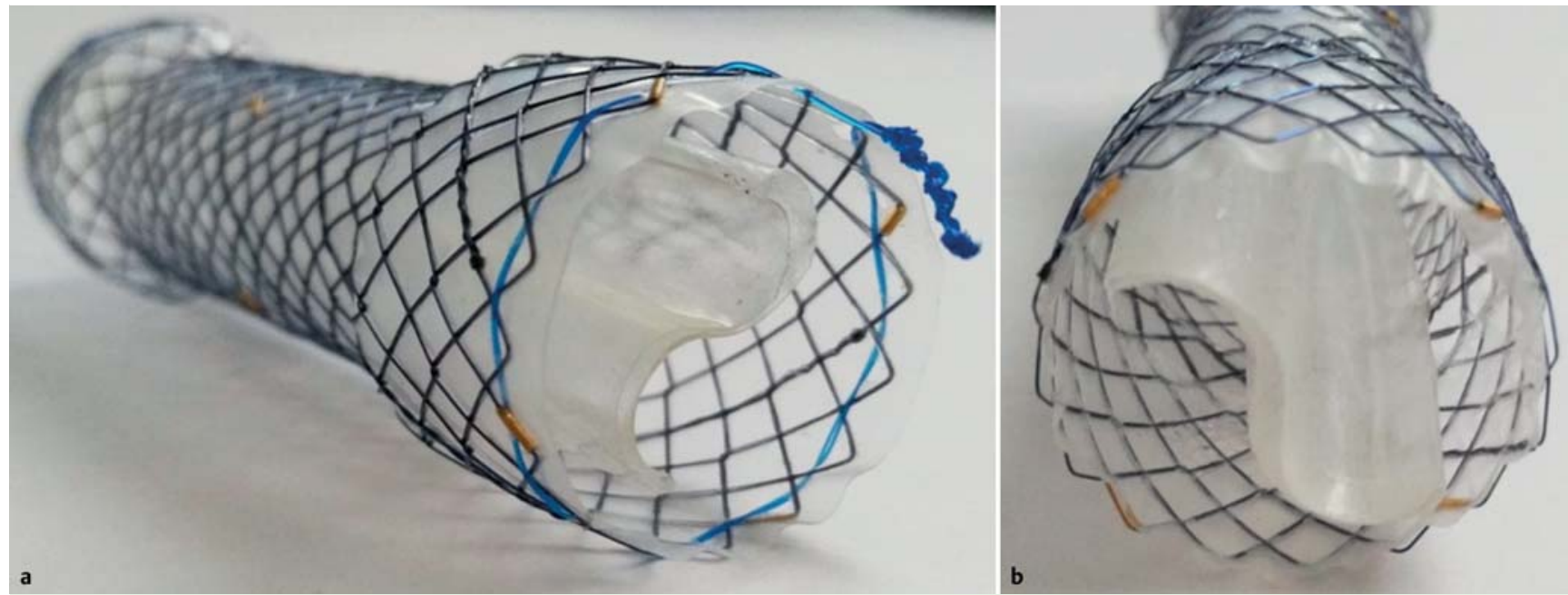

Fig. 1 Antireflux stent. (a) Profile view and (b) En-face view of the internal antireflux valve.

risk of migration and second, it favous the occurrence of gastroesophageal reflux through the stent. Indeed, Valbuena et al. have demonstrated that significant gastro-esophageal reflux occurred in patients with trans-cardial stents [8]. In addition, severe heartburn and respiratory adverse events (AEs) have been reported in approximately $30 \%$ of patients when a SEMS was placed in this location. In order to avoid such complications, stents with an inbuilt antireflux system were proposed more than 30 years ago $[8$, 9]. However, despite major advances in therapeutic endoscopy over the last decade, few high-quality studies concerning such antireflux stents have succeeded in showing a potential benefit in terms of AE prevention and palliation of dysphagia. However, interpretation of these results is limited by the small number of patients included, the heterogeneity of systems used, and the lack of objective parameters assessing antireflux efficacy [9-15]. Therefore, other randomized-controlled studies are needed to assess the efficacy of novel antireflux stents in cancer of the distal esophagus. In addition, the therapeutic gain of proton pump inhibitor (PPI) therapy and postural advice remains to be determined in this situation.

Therefore, our aims were to: 1) evaluate in vivo the mechanical efficacy of an antireflux valve; and 2) compare the clinical results obtained with this antireflux stent with a strategy combining a conventional stent plus PPI therapy and postural advice, in patients with unresectable distal oesophageal carcinoma.

\section{Patients and methods}

$\nabla$

\section{Study design}

Patients with dysphagia from inoperable carcinoma of the distal esophagus or of the gastric cardia were randomly allocated to 2 different arms: 1) placement of an antireflux stent (group 1) with no PPI or postural advice or 2) placement of a standard stent without antireflux valve but associated with PPI therapy and postural advice (group 2). In particular, in group 2, standard doses of PPI (omeprazole $20 \mathrm{mg} /$ day or lansoprazole $30 \mathrm{mg} /$ day) were prescribed and patients were asked to avoid post-prandial rest or tight clothing and to raise their bed head and were systematically prescribed a standard dose of PPIs. In contrast, no specific advice was delivered to group 1 patients.
The randomization process was conducted with sealed envelopes, containing information about the type of stent to be used. Patients were blinded to the type of stent received.

\section{Patients}

All patients between ages 18 and 90 years with a diagnosis of dysphagia due to inoperable carcinoma of the distal esophagus or of the gastric cardia were considered for inclusion in the study. Exclusion criteria were as follows: advanced cancer with life expectancy $<6$ weeks, non-cardia gastric malignancy, symptomatic paralysis of the laryngeal nerve with the risk of swallowing disorder, portal hypertension or coagulation disorders, history of esophagogastric surgery, or impossibility of follow-up on the patient. This study was approved by the Regional Protection of Persons Consultative Comity in Biomedical Research and was in accordance with the 23th of January 1990 law and the Helsinki declaration. Informed oral and written consent were obtained from all patients.

\section{SEMS characteristics}

The antireflux stent (Dostent ${ }^{\circledR}$, M. I. Tech co. LTD, Seoul, Korea) was specifically designed with an internal valve at its distal end, consisting of a soft circumferential membrane ( $\bullet$ Fig. 1 ). The conventional stent $\left(\right.$ Choostent $^{\circledR}$, M. I. Tech co. LTD, Seoul, Korea) had no antireflux system but otherwise had the same characteristics as the antireflux stent. Both were self-expanding metallic stents, $18 \mathrm{~mm}$ in diameter, nitinol composition covered with an external polyurethane membrane anti-migration flares at both ends (24 $\mathrm{mm}$ at the upper side, $30 \mathrm{~mm}$ at the lower side). Stent length ranged from 80 to $170 \mathrm{~mm}$. A retrieval lasso allowed grasping and repositioning or removal of the stent if necessary.

\section{Endoscopic procedure}

All procedures were performed under general anesthesia by experienced endoscopists. First, a pre-therapeutic endoscopy allowed macroscopic visualization of the proximal side of the tumor and endoscopic injection of contrast agent into the stricture. Then, external opaque markers were placed to allow both location of tumor ends under fluoroscopy and choice of stent size. Finally, the stent was placed over a soft guidewire, and gradually deployed inside the malignant stenosis with at least $2 \mathrm{~cm}$ free margin at both ends. In selected cases, a preemptive dilatation 


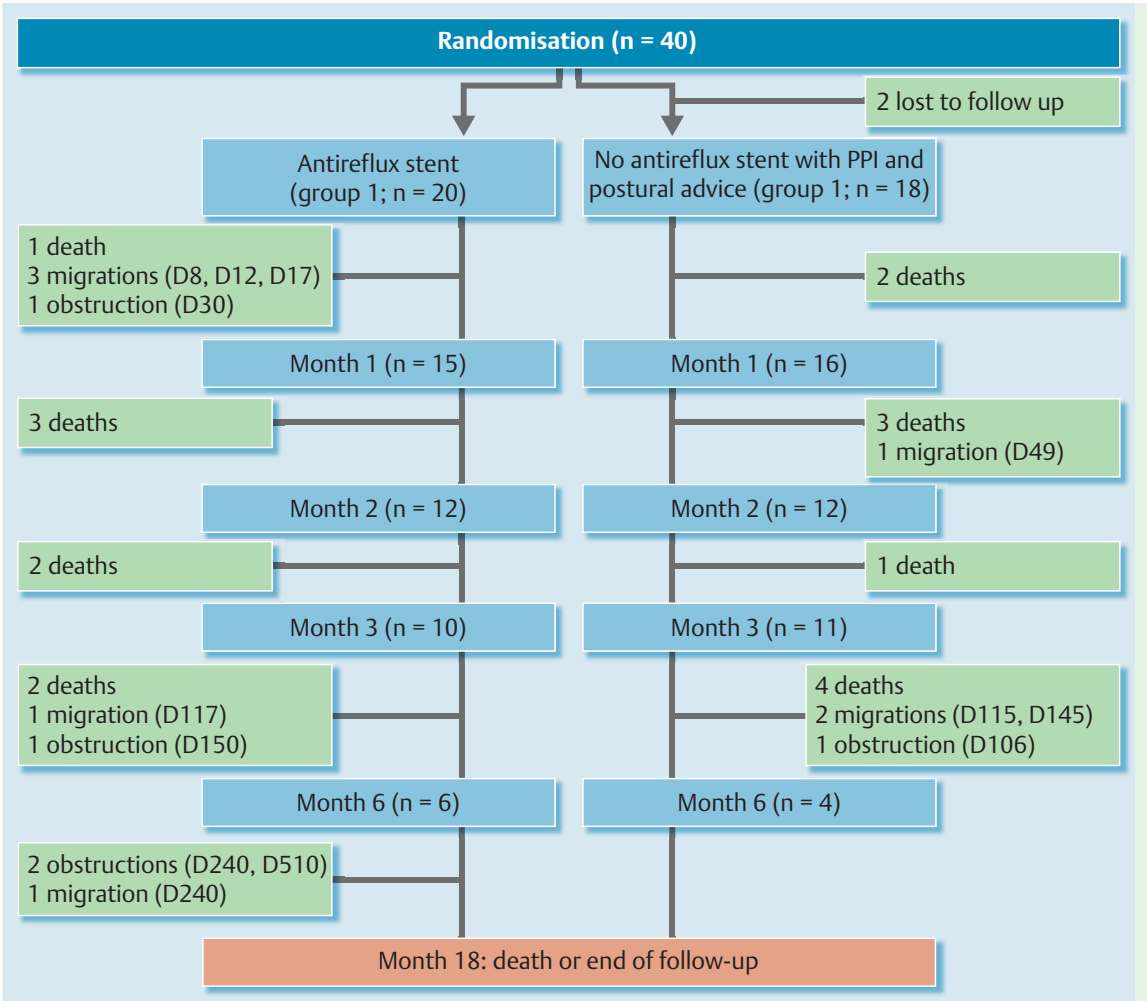

Fig. 2 Flow diagram.

\begin{tabular}{|c|c|c|c|c|}
\hline Trendelenburg position & No reflux & Intra-prosthetic reflux & Sus-prosthetic reflux & Pharyngeal reflux \\
\hline 0 & 0 & 1 & 2 & 3 \\
\hline 5 & 0 & 1 & 2 & 3 \\
\hline 10 & 0 & 1 & 2 & 3 \\
\hline
\end{tabular}

Table 1 Radiological score.

could be performed using either bougienage or balloon dilatation, at the discretion of the endoscopist. The final stent location was controlled by endoscopy and/or fluoroscopy.

\section{Study outcome and follow-up}

The primary endpoint was evaluation of the mechanical efficacy of the antireflux stent, based on a quantitative radiological assessment. At day 2 after the endoscopic procedure, the patient underwent a Trendelenburg maneuver $\left(0^{\circ}, 5^{\circ}, 10^{\circ}\right)$ following ingestion of $0.5 \mathrm{~L}$ liquid barium. A radiological score (ranging from 0 to 9) was calculated according to the intensity of reflux for each position ( Table 1 ). The radiologist interpreting the images was blinded to the type of stent received.

Secondary endpoints included regurgitation, dysphagia, quality of life (QoL) and Organisation Mondiale de la Santé (OMS) scores. All parameters were assessed at baseline (i.e at the time of inclusion in the study) and at 1, 2, 3 and 6 months after placement of the stent. Regurgitation was scored from 0 (none) to 16 (severe). Dysphagia was evaluated using the Atkinson score, which was graded from $0=$ no dysphagia to $4=$ complete dysphagia. QoL was evaluated using the SRQ (Simplified Reflux Qual) ranging from 0 to $100.0 M S$ was scored from 0 (excellent) to 4 (patient confined to bed $>50 \%$ of time). All evaluations were performed through outpatient consultations or regular follow-up by phone contact with the patient and/or the primary care physician by a research nurse or the endoscopist, neither of whom were blinded to the type of stent received.

\section{Statistical analyses}

The radiological reflux score was calculated by adding each result from different Trendelenburg positions. Scores were compared between groups with the Wilcoxon test. Dysphagia, quality of life, OMS score and reflux improvement were compared between the two groups at each period using Wilcoxon test. Overall survival rates were estimated using the Kaplan-Meier method. Statistical differences in overall survival were tested by log-rank test. Relative risks (RR) were estimated with their 95\% confidence interval using a Cox model. $P$ values were two sided and statistical significance was accepted at the $P<0.05$ level. SAS Software was used for all statistical analyses.

\section{Results \\ $\nabla$}

\section{Patients and procedures}

Over a 2-year period, 40 patients were included in nine French university hospitals (Nantes, Rouen, Limoges, Poitiers, Strasbourg, Mulhouse, Grenoble, Marseille and Paris Cochin). Twenty patients were allocated to group 1 and 20 to group 2. Two patients were lost to follow up shortly after inclusion in group 2, and were therefore excluded from the study ( $\bullet$ Fig.2). Patients and tumor characteristics are presented in Table2. No statistical differences were noted between the groups in terms of patient or tumor characteristics or stent size. Stent insertions were technically successful in all patients. 


\begin{tabular}{|c|c|c|c|}
\hline & $\begin{array}{l}\text { Antireflux stent } \\
\text { (Group } 1 ; n=20 \text { ) }\end{array}$ & $\begin{array}{l}\text { Conventional stent plus PPI/ } \\
\text { postural advice (Group 2; } n=18 \text { ) }\end{array}$ & $P$ value \\
\hline Age (years) (mean [SD]) & $68.9[11.1]$ & $74.1[12.1]$ & $P=0.12$ \\
\hline \multicolumn{4}{|l|}{ Gender } \\
\hline Male (\%) & $16(80.0)$ & $15(83.3)$ & \multirow{2}{*}{$P=1$} \\
\hline Female (\%) & $4(20.0)$ & $3(16.7)$ & \\
\hline \multicolumn{4}{|l|}{ Tumor histopathology (\%) } \\
\hline Squamous cell carcinoma & $9(45)$ & $7(38.9)$ & \multirow{3}{*}{$P=0.86$} \\
\hline Adenocarcinoma & $10(50.0)$ & $11(61.1)$ & \\
\hline Undifferentiated & $1(5.0)$ & $0(0)$ & \\
\hline \multicolumn{4}{|l|}{ General extension (\%) } \\
\hline No & $8(40.0)$ & $10(55.6)$ & \multirow{2}{*}{$P=0.34$} \\
\hline Yes & $12(60.0)$ & $8(44.4)$ & \\
\hline Tumor size (mean [SD])(cm) & $6.9[3.0]$ & $6.7[1.8]$ & $P=1$ \\
\hline \multicolumn{4}{|l|}{ Preemptive dilatation (\%) } \\
\hline No & $13(65.0)$ & $13(72.2)$ & \multirow{2}{*}{$P=63$} \\
\hline Yes & $7(35.0)$ & $5(27.8)$ & \\
\hline
\end{tabular}

Table 2 Patients characteristics.

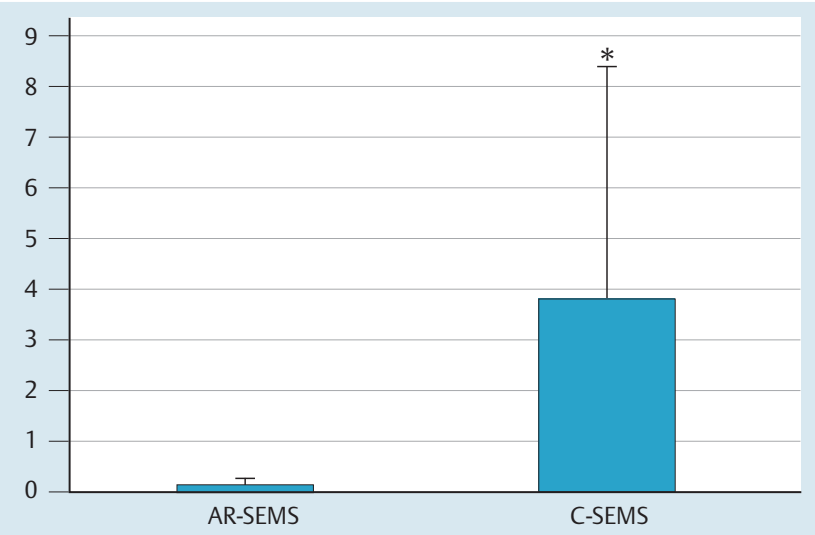

Fig. 3 Radiological reflux score assessing trans-prosthetic reflux during a Trendelenburg maneuver. The antireflux valve self-expanding metal stent (group 1) showed clear prevention of radiological reflux as compared to the conventional self-expanding metal stent (group 2) $(P<0.0001)$

\section{Outcomes}

Regarding our primary endpoint, the radiological score was significantly lower in group 1 than in group 2 (0.7 vs 5.3, $P<$ 0.0001)( Fig.3). No statistical difference was found in terms of overall mortality ( $\bullet$ Fig. 4$)$

The regurgitation score was significantly lower in group 1 than in group 2 at 2 months after stent placement $(P=0.03)$. However, it was not statistically different at 1,3 and 6 months ( Fig.5a). There were no difference between the two groups in terms of dysphagia, QoL or OMS scores ( $\bullet$ Fig. 5 b, c, d). No statistical difference was found in terms of overall mortality. However, a tendency toward longer survival was noted group 1 (median [95\% CI]): 242 [108 - 390] vs 165 [60 - 215] days; $P=0.57$ ). Pre-emptive dilatation was the only parameter statistically associated with longer life expectancy $(R R=2.44[1.05-5.72] P=0.0393)$.

\section{Adverse events}

No death, bleeding or perforation occurred during the procedures. One patient in group 1 had a severe aspiration due to gastroesophageal reflux during the radiological test at day 2 after stent placement. The major cause of death during the follow-up was cancer evolution in 26 (68\%) cases, including esophageal cancer growth, carcinomatous meningitis or pleurisy. One pa-

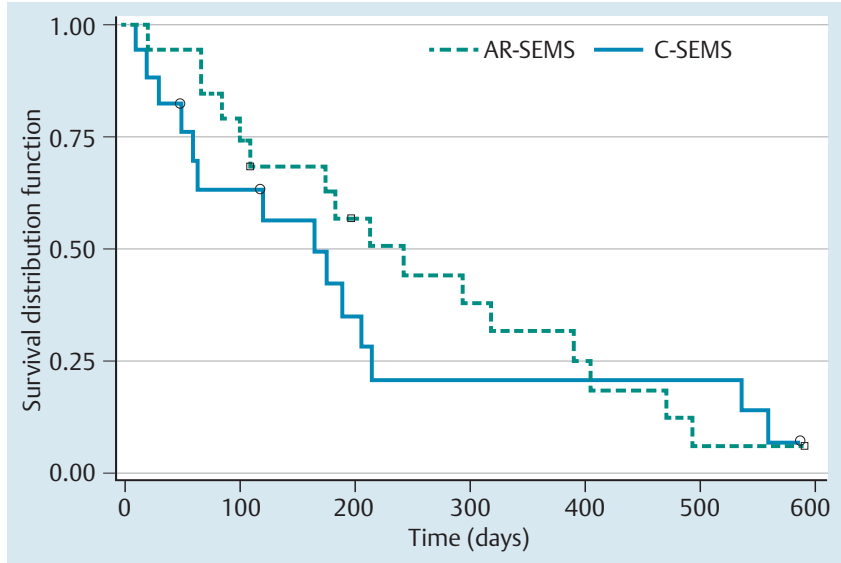

Fig.4 Overall survival curves showing no difference between the two different strategies, i.e. antireflux stent alone (group 1) versus conventional stent associated with PPIs (group 2).

tient in group 1 died from hematemesis 20 months after stent placement. Two patients died from pneumoniae at 1 and 9 months after SEMS placement in group 1 and group 2, respectively.

A total of five stent migrations occurred in group 1 on days 8,12 , 17,117 and 240 after stent placement, respectively. Three migrations were reported in group 2 on days 49, 115 and 145 after stent placement, respectively. No significant difference was observed between the two groups in terms of migrations $(P=0.41)$. In addition, four stent obstructions were observed in group 1 while only one stent obstruction was reported in group 2.Considering migrations and obstructions together, more AEs were observed in the group 1 than in group 2 (55\% versus $18 \%$; $P=$ 0.0196). Neither severe retrosternal pain leading to the stent removal nor sepsis related to the stent insertion was observed. 

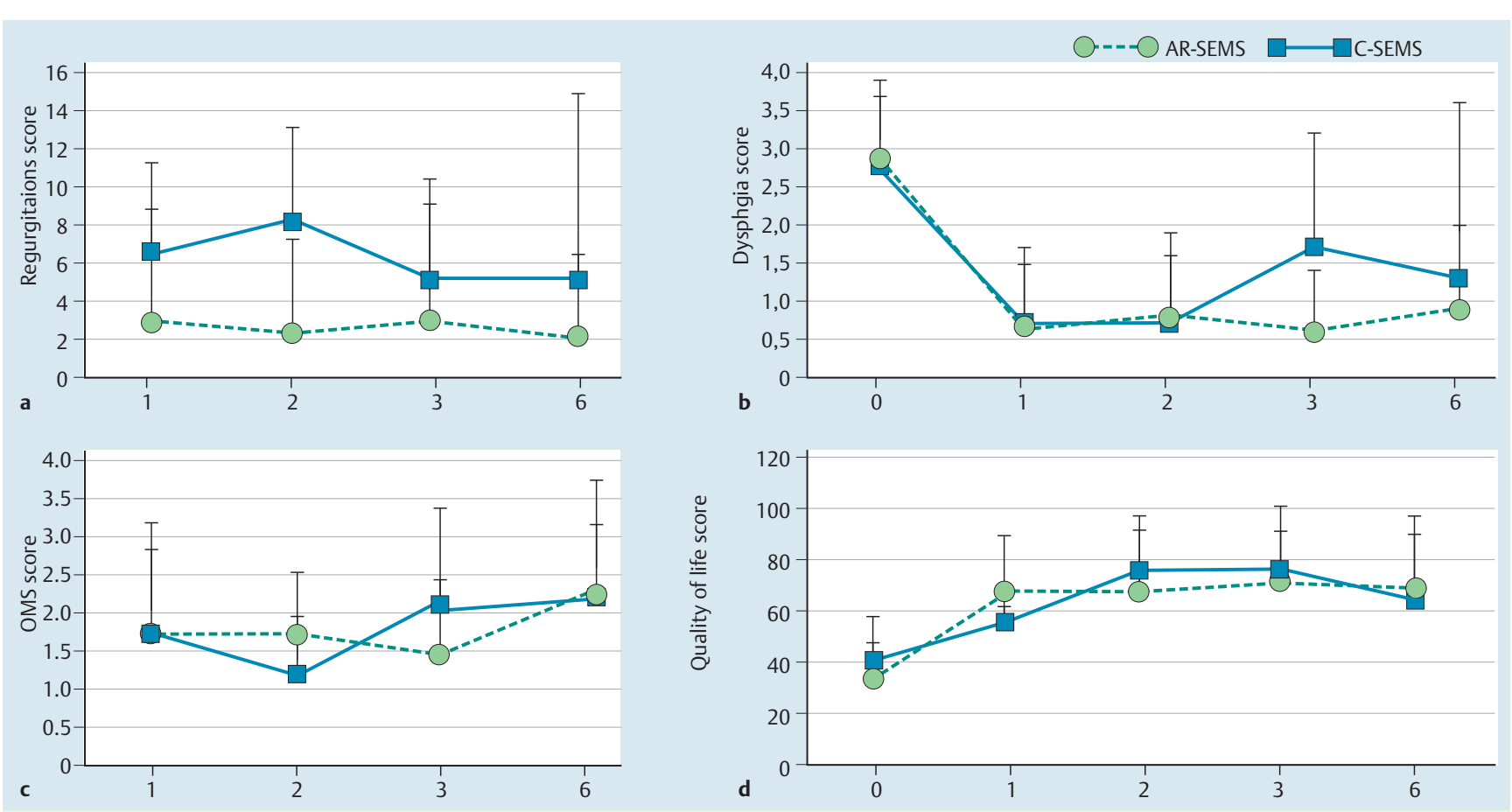

Fig. 5 a regurgitation scores $\mathbf{b}$ dysphagia c Organisation Mondiale de la Santé (OMS) scores and $\mathbf{d}$ quality of life scores. No difference was noted between patients with antireflux stent alone (group 1) versus patients with conventional stent associated with PPIs (group 2).

\section{Discussion}

$\nabla$

SEMS have been shown to be safe and effective in palliation of dysphagia in lower esophageal and esophagogastric junction cancers $[4,7]$. However, the use of stents can predispose to gastro-esophageal reflux due to the disappearance of physiologic barrier, resulting in impaired QoL for patients [8]. Reflux may even cause severe complications such as aspiration and decrease life expectancy. In addition, pain and discomfort are the main issues in palliative situations, emphasizing the need for a strongly positive benefit/risk balance. Therefore, development of novel stents effective in preventing reflux and its complication would represent major progress in advanced esophageal cancer. However, while various stents have been tested during the last decade, none of them have shown real clear benefit in terms of reflux prevention [16]. Furthermore, some of the stents with an inbuilt antireflux system showed the same rate of obstruction but a higher rate of migration than standard ones [13].

Our randomized, controlled study demonstrated that antireflux stents have clear mechanical efficacy based on radiological examination. Indeed, we observed a striking difference in terms of barium refluxate at day 2 between the 2 groups. This finding was based on a rigorous radiological procedure which contained a Trendelenburg maneuver. In addition, the images were independently interpreted by a radiologist who was blinded to the type of stent received. This is clearly original since no other study has directly assessed the efficacy of an antireflux valve with objective measurements of radiological reflux. Indeed, most studies on stents assessed subjective parameters such as GERD questionnaires, and only one randomized study used pH-metry to demonstrate quantitative improvement by antireflux stents [14], which is consistent with our findings.

In addition, our study showed that antireflux stents were as effective for symptom control as conventional stents combined with PPI therapy and postural advice. Moreover, the regurgitation score at 2 months was superior in group 1 as compared with group 2.The overall lack of statistical significance between groups in our study is in contrast with three other studies showing superiority of antireflux stents over conventional stents on GERD symptoms and QoL $[13,14,17]$. There are several potential explanations for this: First, we did not directly compare the clinical efficacy of two types of stents but of two different strategies. Indeed, while group 1 patients only received the antireflux stent, group 2 patients were also prescribed PPI therapy and were asked to follow dietary and postural advice. Our results suggest that antireflux stents are as effective as this latter strategy in preventing clinical manifestations of GERD. This is of importance since PPI therapy is costly and in some cases, it can be difficult to educate patients. However, few data are currently available on the impact of patient education and GERD pharmacological treatment on palliation of esophageal cancer [18]. Second, the lack of statistical significance between the 2 groups in terms of clinical parameters might be due to the small sample size of the 2 groups, especially during the follow up of these patients with advanced cancers. Third, we cannot rule out the possibility that the design of the stent was associated with radiological efficacy but not with clinical efficacy, since other randomized studies reported the absence of difference between various antireflux and conventional stents on symptoms $[10,11,12,15]$. However, these results must be interpreted with caution due to the variety of types of stents and procedures, and the clear radiological efficacy of antireflux stents in our study favors clinical efficacy.

Migration or obstruction of stents is an important issue in the management of patients during the course of the disease. In our study, we reported significantly more AEs with antireflux stents as compared to conventional stents. Indeed, more obstructions were noted in the antireflux system group than in the standard stent group. However, the rate of migration did not differ between the groups. These results need to be interpreted with caution since the study was not designed to specifically address this 
issue. However, it is surprising to note that the rate of AEs with antireflux stents and conventional stents seems to be respectively higher and lower than the ones reported in other studies. For instance, Blomberg et al. reported a complication rate ranging from $35 \%$ to $43 \%$ with various types of stents [10] while our complication rate ranged from $18 \%$ with conventional stents to $55 \%$ with antireflux stents. In contrast, our rate of stent obstruction was $35 \%$, which was slightly higher than the one reported by Sabharwal et al. [13] This might be due to the longer follow up in our study, as suggested by the fact that most AEs occurred after day 100. Also, differences between $\mathrm{AE}$ rates might be related either to the design of the stent or to the endoscopist. In our multicenter study, we did not specifically assess the level of expertise of endoscopists, which might be an important bias. However, all procedures were performed in tertiary-referral centers. In addition and in contrast with other studies reporting severe complications such as gastric or esophageal perforations, we did not observe any severe AEs related to the stent insertion procedure. Also, no stent migration occurred in patients undergoing bougienage during insertion, except in one patient who had stent migration 8 days after the preemptive dilation. Recent studies have reported promising results using newly designed stents with double layers or external flanges to prevent migration [19]. Also, Mudumbi et al. have proposed anchoring standard stents with large over-the-scope clips, including instructions for subsequent removal if necessary [20]. However, such studies on newly designed stents or anchoring strategies are, to date, limited to proof of concept and warrant further evaluation using randomized controlled trials.

Our study has several strengths. First, it is a randomized controlled study, with patients and the radiologist performing the reflux evaluation being blinded to the type of stent received. Second, the multicenter design and type of patient included reflect "real-life" conditions. Third, an important strength of our study was its relatively long-term follow up. Indeed, the median follow up was 7.5 months, which is superior to most studies previously published. One-third of our patients died before the end of the study, which allowed us to perform an ancillary analysis on predictive factors of death. However, neither the size of tumor, type of stent or occurrence of complications was predictive of shorter life expectancy. This is, of course, limited by the small sample size of the study.

Our study also has important limits. First, the sample size is relatively small, limiting the possibility of thoroughly evaluating key parameters such as survival, QoL or symptom relief rates. Second, compliance with PPI treatment and postural advice was not evaluated in this study. Also, potential self-administration of antacid medications in patients with the antireflux stent (group 1) might constitute an important bias. However, our study and others [8$16,21]$ showed that gastric regurgitation can occur even under medical treatment after stent placement, to include food or bile reflux. Therefore, we believe that potential medication biases are probably less important than the mechanical effect of the stent. Third, questionnaires used to assess regurgitation, dysphagia, And QoL OMS scores were completed by research nurses or physicians who were not blinded to the type of stent received, which is an important limitation. Last, this study was designed to compare two different strategies rather than two different stents. Therefore, we cannot draw conclusions about the direct effect of the antireflux valve on gastrointestinal symptoms and patient QoL. Nevertheless, we think that such study comparing different stent designs would not be sufficient to draw practical conclusions regarding patients' management, particularly regarding the need for PPI therapy and postural advice.

In conclusion, our study demonstrated that antireflux stents are not only more efficient for preventing trans-prosthetic reflux, but are also as effective for relieving symptoms and improving QoL as a strategy that combines conventional stents with PPI therapy and postural advice. While antireflux stents had a higher rate of AEs, they were minor and easily managed endoscopically. Other treatments such as brachytherapy, external radiotherapy or chemotherapy have also shown promising results in this situation [22-27] and should be further evaluated. Future research should focus on optimal treatment algorithms, including the otential association between endoscopic and non-endoscopic therapies.

\section{Competing interests: None}

\section{Institutions}

${ }^{1}$ Institut des Maladies de l'Appareil Digestif, Centre Hospitalier Universitaire, Nantes cedex, France

${ }^{2}$ CIC-INSERM, Centre Hospitalier Universitaire, Nantes cedex, France

3 Service d'Hépatogastroentérologie, Centre Hospitalier Universitaire, Rouen, France

${ }^{4}$ Service d'Hépatogastroentérologie, Centre Hospitalier Universitaire,

Limoges, France

${ }^{5}$ Service d'Hépatogastroentérologie, Centre Hospitalier Universitaire, Poitiers, France

${ }^{6}$ Service d'Hépatogastroentérologie, Centre Hospitalier Universitaire,

Strasbourg, France

${ }^{7}$ Département de Biostatistiques, Centre Hospitalier Universitaire, Nantes, France

${ }^{8}$ Service d'Hépatogastroentérologie, Centre Hospitalier Universitaire, Mulhouse, France

9 Service d'Hépatogastroentérologie, Centre Hospitalier Universitaire, Grenoble, France

${ }^{10}$ Service d'Hépatogastroentérologie, Hopital Saint-Joseph, Marseille, France

11 Service d'Hépatogastroentérologie, Hopital Cochin, Paris, France

\section{Acknowledgements}

$\nabla$

The authors acknowledge the French Society of Digestive Endoscopy which supported the study. The authors acknowledge Mrs Françoise Robin in Lyon for her logistical support regarding coordination of the study, as well as endoscopy-specialized nurses in all centers involved in this work. The authors also thank Dr. Jeremy Brégeon for his support to the edition of the manuscript.

\section{References}

1 Bouvier AM, Binquet C, Gagnaire A et al. Management and prognosis of esophageal cancers: has progress been made? Eur J cancer 2006; 42: $228-233$

2 Siersema PD. New developments in palliative therapy. Best Pract Res Clin Gastroenterol 2006; 20: 959-978

3 Stein HJ, Siewert JR. Improved prognosis of resected esophageal cancer. World J Surg 2004; 28: 520-525

4 Martin RC, Cannon RM, Brown RE et al. Evaluation of quality of life following placement of self-expanding plastic stents as a bridge to surgery in patients receiving neoadjuvant therapy for esophageal cancer. Oncologist 2014; 19: 259-265

5 Wenger $U$, Luo J, Lundell $L$ et al. A nationwide study of the use of selfexpanding stents in patients with esophageal cancer in Sweden. Endoscopy 2005; 37: 329-334

6 Knyrim K, Wagner HJ, Bethge $N$ et al. A controlled trial of an expansive metal stent for palliation of esophageal obstruction due to inoperable cancer. N Engl J Med 1993; 329: 1302 - 1307

7 Ell C, May A. Self-expanding metal stents for palliation of stenosing tumors of the esophagus and cardia: a critical review. Endoscopy 1997; 29: $392-398$

8 Valbuena J. Endoscopic palliative treatment of esophageal and cardial cancer: a new antireflux prosthesis: a study of 40 cases. Cancer 1984; 53: $993-998$ 
9 Dua KS, Kozarek R, Kim J et al. Self-expanding metal esophageal stent with anti-reflux mechanism. Gastrointest Endosc 2001; 53: 603-13

10 Blomberg J, Wenger $U$, Lagergren J et al. Antireflux stent versus conventional stent in the palliation of distal esophageal cancer. A randomized, multicenter clinical Scand. J Gastroenterol 2010; 45: 208 - 216

11 Nunes C, Waechter F, Sampaio J et al. Comparative post-operative study of prostheses, with and without an anti-reflux valve system, in the palliative treatment of esophageal carcinoma. Hepatogastroenterology 1999; 46: 2859-2864

12 Shim C, Jung I, Cheon Y et al. Management of malignant stricture of the esophagogastric junction with a newly designed self-expanding metal stent with an antireflux mechanism. Endoscopy 2005; 37: 335-339

13 Sabharwal T, Gulati MS, Fotiadis $N$ et al. Randomised comparison of the FerX Ella antireflux stent and the ultraflex stent: proton pump inhibitor combination for prevention of post-stent reflux in patients with esophageal carcinoma involving the esophago-gastric junction. J Gastroenterol Hepatol 2008; 23: $723-728$

14 Power C, Byrne PJ, Lim K et al. Superiority of anti-reflux stent compared with conventional stents in the palliative management of patients with cancer of the lower esophagus and esophago-gastric junction: results of a randomized clinical trial. Dis Esophagus 2007; 20: 466-470

15 Schoppmeyer K, Golsong J, Schiefke I et al. Antireflux stents for palliation of malignant esophagocardial stenosis. Dis Esophagus 2007; 20: 8993

16 Sgourakis G, Gockel I, Radtke A et al. The use of self-expanding stents in esophageal and gastroesophageal junction cancer palliation: a meta-analysis and meta-regression analysis of outcomes. Dig Dis Sci 2010; 55: $3018-3030$

17 Osugi $H$, Lee S, Higashino $M$ et al. Usefulness of self-expandable metallic stent with an antireflux mechanism as a palliation for malignant strictures at the gastroesophageal junction. Surg Endosc 2002; 16: 14781482

18 Sagar PM, Gauperaa T, Sue-Ling $H$ et al. An audit of the treatment of cancer of the oesophagus. Gut 1994; 35: $941-945$
19 Walter D, van den Berg $M$, van Hooft J et al. A new fully covered metal stent with anti-migration. Endoscopy 2014; 46: $1101-1105$

20 Mudumbi S, Velazquez-Aviña S, Neumann $H$ et al. Anchoring of self-expanding metal stents using the over-the-scope clip, and a technique for subsequent removal. Endoscopy 2014; 46: 1106-1109

21 Christie NA, Buenaventura PO, Fernando HC et al. Results of expandable metal stents for malignant esophageal obstruction in 100 patients : short term and long term follow up. Ann Thor Surg 2001; 71: 1797 1802

22 Gebski V, Burmeister B, Smithers BM et al. Survival benefits from neoadjuvant chemoradiotherapy or chemotherapy in oesophageal carcinoma: a meta-analysis. Lancet Oncol 2007; 8: 226-234

23 Taieb J, Mitry E, Boige Vet al. Optimization of 5-fluorouracil (5-FU)/cisplatin combination chemotherapy with a new schedule of leucovorin, 5-FU and cisplatin (LV5FU2-P regimen) in patients with biliary tract carcinoma. Ann Oncol 2002; 13: 1192 - 1196

24 Homs $M$, Steyerberg E, Eijkenboom $W$ et al. Single-dose brachytherapy versus metal stent placement for the palliation of dysphagia from oesophageal cancer: multicentre randomised trial. Lancet 2004; 364: $1497-1504$

25 Zhu HD, Guo JH, Mao AW et al. Conventional stents versus stents loaded with (125)iodine seeds for the treatment of unresectable oesophageal cancer: a multicentre, randomised phase 3 trial. Lancet Oncol 2014; 15: $612-619$

26 Hirdes MM, van Hooft JE, Wijrdeman HK et al. Combination of biodegradable stent placement and single-dose brachytherapy is associated with an unacceptably high complication rate in the treatment of dysphagia from esophageal cancer. Gastrointest Endosc 2012; 76: 267 274

27 Touchefeu Y, Archambeaud I, Landi B et al. Chemotherapy versus selfexpanding metal stent as primary treatment of severe dysphagia from unresectable oesophageal or gastro-oesophageal junction cancer. Dig Liver Dis 2014; 46: 283 - 286 